\title{
Eigenparameter Dependent Inverse Sturm-Liouville Problems
}

\author{
C. Maeve McCarthy ${ }^{1, *}$ and William Rundell ${ }^{2}$ \\ ${ }^{1}$ Department of Mathematics and Statistics, Murray State \\ University, Murray, USA \\ ${ }^{2}$ Department of Mathematics, Texas A \& M \\ University, Texas, USA
}

\begin{abstract}
Uniqueness of and numerical techniques for the inverse Sturm-Liouville problem with eigenparameter dependent boundary conditions will be discussed. We will use a Gel'fand-Levitan technique to show that the potential $q$ in

$$
\begin{aligned}
& -u^{\prime \prime}+q u=\lambda u, \quad 0<x<1 \\
& u(0)=0, \\
& (a \lambda+b) u(1)=(c \lambda+d) u^{\prime}(1)
\end{aligned}
$$

can be uniquely determined using spectral data. In the presence of finite spectral data, $q$ can be reconstructed using a successive approximation method that involves solving a hyperbolic boundary value problem that arises in the the Gel'fand-Levitan analysis. We also consider a shooting method where the right endpoint boundary condition is used in conjunction with a quasi-Newton scheme to recover the unknown potential, $q$.
\end{abstract}

Key Words: Inverse spectral theory; Eigenparameter dependent Sturm-Liouville problem; Gel'fand-Levitan method; Quasi-Newton method.

*Correspondence: C. Maeve McCarthy, Department of Mathematics and Statistics, Murray State University, Murray, KY 42071, USA. 


\section{INTRODUCTION}

The direct eigenparameter dependent problem under consideration involves solving the differential equation

$$
-u^{\prime \prime}+q u=\lambda u, \quad 0<x<1
$$

subject to boundary conditions

$$
\begin{aligned}
& u(0)=0 \\
& (a \lambda+b) u(1)=(c \lambda+d) u^{\prime}(1)
\end{aligned}
$$

with $\delta=a d-b c>0$.

An essential difference between the regular Sturm-Liouville problem and the eigenparameter dependent one is that the latter may have one more eigenvalue than the former. For the regular problem, the eigenvalues are solutions of a transcendental equation of the form

$$
\cot \theta(\lambda, 1)=C
$$

where $\theta(\lambda, x)$ is the Prüfer angle and $C$ is a constant depending on the boundary conditions at the right end-point. Each branch of the graph of cotangent yields precisely one eigenvalue. When the boundary conditions contain the eigenvalue as in Eq. (3) the transcendental has the form

$$
\cot \theta(\lambda, 1)=\frac{a \lambda+b}{c \lambda+d} .
$$

One of the branches of the graph of cotangent yields two eigenvalues while the others yield one. This extra eigenvalue impacts the asymptotics (c.f. Appendix).

The appearance of the eigenvalue in the boundary condition also causes the Sturm-Liouville operator to lose its self-adjointness in $L^{2}[0,1]$. In fact, the eigenfunctions do not form a basis in $L^{2}[0,1]$ unless one of the eigenfunctions is removed from the set. It is possible, however, to introduce an associated operator in $L^{2}[0,1] \oplus \mathbb{C}$ that is self-adjoint. The analysis of the applications, completeness, and expansion theory of the regular direct problem is found in the work of Walter (1973) and Fulton (1977). Binding et al. (1993) present asymptotic, oscillation, and comparison results.

Uniqueness results for the inverse spectral problem for Sturm-Liouville equations of this type have been studied recently by Browne and Sleeman (1996, 1997), and by Binding et al. (2000). In (Browne and Sleeman, 1996) Browne and Sleeman discuss uniqueness for nodal spectral data. In Browne and Sleeman (1997), they establish uniqueness when $p=r=1$, given one spectrum and a sequence of norming constants. A more general result is that of Binding et al. (2000), which establishes uniqueness results for the cases when (i) two spectra are prescribed, (ii) one spectrum and a sequence of norming constants are prescribed, and (iii) one spectrum with even $p, q, r$ are prescribed. Case (ii) is addressed again in Binding et al. (2002), where Binding et al. construct a mapping from the eigenparameter dependent SturmLiouville problem to the regular Sturm-Liouville and apply the known results for the inverse Sturm-Liouville problem. 
We reconsider here, the inverse problem where two spectra are prescribed. More precisely, we seek to recover the unknown potential $q \in L^{2}(0,1)$ from two sequences of eigenvalues $\left\{\lambda_{n}^{i}\right\}_{n=1}^{\infty}$ and their associated boundary condition constants $a_{i}, b_{i}, c_{i}, d_{i}$, for $i=1,2$ where

$$
\begin{array}{lll}
-u^{\prime \prime}+q u=\lambda_{n}^{1} u, & u(0)=0, & \left(a_{1} \lambda_{n}^{1}+b_{1}\right) u(1)=\left(c_{1} \lambda_{n}^{1}+d_{1}\right) u^{\prime}(1) \\
-u^{\prime \prime}+q u=\lambda_{n}^{2} u, & u(0)=0, & \left(a_{2} \lambda_{n}^{2}+b_{2}\right) u(1)=\left(c_{2} \lambda_{n}^{2}+d_{2}\right) u^{\prime}(1) .
\end{array}
$$

Although previous results establish uniqueness, they do not provide constructive algorithms for the solution of the eigenparameter dependent problem. In practice, one cannot measure an infinite spectrum experimentally. However, given a finite set of spectral data, of size $N$, we expect to be able to recover a potential $q^{N}$, that gives rise to the same set of spectral data when the direct problem is solved.

In Sec. II, we provide a proof of uniqueness based on a Gel'fand-Levitan argument. We develop two algorithms for the reconstruction of $q$ based on finite data in Secs. III and IV. Our first algorithm is based on the conversion of the spectral inverse problem into an overdetermined boundary value problem for a hyperbolic operator which can be solved iteratively. This approach is based on the work of Rundell and Sacks (1992). Our second algorithm is based on the combination of techniques from differential equations and finite-dimensional optimization. Using a shootingmethod, the solution of an appropriate initial value problem is forced to satisfy the right endpoint boundary condition and a quasi-Newton scheme to recover the unknown potential $q$. This is an extension of the work of Lowe et al. (1992). Finally, we present a discussion of the implementation and results of both algorithms in Sec. V.

\section{UNIQUENESS OF THE INVERSE PROBLEM}

We use a Gel'fand-Levitan approach to establish uniqueness of the inverse problem. For a given value of $\lambda$ let $u(x ; q, \lambda)$ represent the solution to the initial value problem

$$
\mathcal{L}_{q} u:=-u^{\prime \prime}+q(x) u=\lambda u, \quad u(0)=0, \quad u^{\prime}(0)=1
$$

For $f \in L^{2}[0,1]$ and $z$ complex, we define the eigenfunction transform of $f$ by

$$
\mathcal{S}_{q}[f](z):=\int_{0}^{1} f(t) u(t ; q ; z) d t
$$

This operator has the following properties:

Lemma II.1. For $f \in L^{2}[0,1], \mathcal{S}_{q}[f](z)$ is analytic of order $1 / 2$ in $z$. $\mathcal{S}_{q}[f](z)=0$ for all real $z$ implies that $f=0$ a.e., and $\lim _{x \rightarrow \infty} \mathcal{S}_{q}[f](x)=0$.

Proof. This follows from some well-known properties of the solution $u(x ; q ; z)$. First, from Pöschel and Trubowtiz (1987, p. 13), $u(x ; q ; z)$ is an analytic function of order $1 / 2$, and so by Levin (1980, p. 387-388) the same is true of $\mathcal{S}_{q}[f]$. Second, if $\int_{0}^{1} f(t) u(t ; q ; z) d t=0$ then, in particular, $\int_{0}^{1} f(t) u\left(t ; q ; \mu_{n}\right) d t=0$ when $\mu_{n}$ are the 
Dirichlet eigenvalues of the operator $\mathcal{L}$, that is, when $z=\mu_{n}$ and $u\left(1 ; q ; \mu_{n}\right)=0$. However, $\left\{u\left(t ; q ; \mu_{n}\right)\right\}_{n=1}^{\infty}$ is a complete basis in $L^{2}[0,1]$ and so it follows that $f=0$. The final statement follows from the asymptotic formula for $u(t ; q ; z)$

$$
u(t ; q ; z)=\frac{\sin z t}{z}+O\left(\frac{e^{\Im(z) t}}{z}\right)
$$

and the fact that $\left\{u\left(t ; q ; \mu_{n}\right)\right\}$ is a basis with norm uniformly bounded in $n$.

For real $a_{i}, b_{i}, c_{i}, d_{i}$, we define

$$
\theta_{i}(z)=\frac{a_{i} z+b_{i}}{c_{i} z+d_{i}} \quad i=1,2 .
$$

so that the right hand boundary conditions of Eqs. (4) and (5) can be written in the equivalent form

$$
u^{\prime}(1 ; q ; \lambda)=\theta_{i}(\lambda) u(1 ; q ; \lambda) .
$$

In order to ensure that we are providing new information from the second eigenvalue sequence we will require that the coefficients in the meromorphic functions $\theta_{i}$ satisfy

$$
\left|\begin{array}{ll}
a_{1} & a_{2} \\
c_{1} & c_{2}
\end{array}\right| \neq 0 \quad \text { or } \quad\left|\begin{array}{ll}
b_{1} & b_{2} \\
d_{1} & d_{2}
\end{array}\right| \neq 0
$$

Note that this condition for uniqueness is weaker than the one given by Binding et al. (2000), namely:

$$
\left|\begin{array}{ll}
a_{1} \lambda+b_{1} & c_{1} \lambda+d_{1} \\
a_{2} \lambda+b_{2} & c_{2} \lambda+d_{2}
\end{array}\right| \neq 0 \quad \text { for all } \lambda \in \mathbb{R} .
$$

We denote by $\left\{\lambda_{n}^{i}\right\}_{n=1}^{\infty}$ the eigenvalues of the Sturm-Liouville problem (6) subject to the boundary condition (9) for $i=1,2$.

Recall the Gel'fand-Levitan representation,

$$
u\left(x ; q_{2}, \lambda\right)=u\left(x ; q_{1}, \lambda\right)+\int_{0}^{x} K\left(x, t ; q_{1}, q_{2}\right) u\left(t ; q_{1}, \lambda\right) d t
$$

where $K\left(x, t ; q_{1}, q_{2}\right)$ satisfies the usual hyperbolic initial value problem and is independent of the parameter $\lambda$,

$$
\begin{aligned}
& K_{t t}-K_{x x}+\left[q_{2}(x)-q_{1}(t)\right] K=0, \quad 0<t<x<1 \\
& K(x, 0)=0, \quad K\left(x, x ; q_{1}, q_{2}\right)=\frac{1}{2} \int_{0}^{x}\left[q_{2}(s)-q_{1}(s)\right] d s .
\end{aligned}
$$

The following lemma is proven by Rundell and Sacks, $(1992, \S 6]$ :

Lemma II.2. If $q_{1}$ and $q_{2}$ are in $L^{2}[0,1]$ and if the associated functions $K\left(x, t ; q_{1}, q_{2}\right)$ satisfy $K\left(1, t ; q_{1}, q_{2}\right)=0$ and $K_{x}\left(1, t ; q_{1}, q_{2}\right)=0$, then $q_{1}=q_{2}$.

For the sake of brevity, we suppress the dependence on $q_{1}$ and $q_{2}$ in $K$. Applying Eq. (9), the boundary condition at $x=1$, to the representation (11) with two 
potentials $q_{1}$ and $q_{2}$ gives

$$
K(1,1) u\left(1, q_{1} ; \lambda\right)+\int_{0}^{1}\left[K_{x}(1, t)-\theta_{i}(\lambda) K(1, t)\right] u\left(t, q_{1} ; \lambda\right) d t=0
$$

for each eigenvalue $\lambda$. In fact, $\lambda$ is an eigenvalue precisely when this equation holds. For $z=\lambda_{n}^{i}$ we rewrite this as

$$
K(1,1) u\left(1 ; q_{1} ; z\right)+\int_{0}^{1}\left[K_{x}(1, t)-\theta_{i}(z) K(1, t)\right] u\left(t ; q_{1} ; z\right) d t=0 .
$$

Multiplying through by $\sqrt{z}$, using the asymptotic form for $u(t ; q ; z)$ in Eq. (7), and applying Lemma II.1, shows that the term involving the integral goes to zero as $z \rightarrow \infty$ for $z$ real, but the first term does not. This implies that $K(1,1)=$ $(1 / 2) \int_{0}^{1}\left[q_{2}(s)-q_{1}(s)\right] d s=0$ and so the mean values of $q_{1}$ and $q_{2}$ must be the same. Hence

$$
\int_{0}^{1}\left[K_{x}(1, t)-\theta_{i}\left(\lambda_{n}^{i}\right) K(1, t)\right] u\left(t ; q_{1} ; \lambda_{n}^{i}\right) d t=0 .
$$

Set

$$
\Phi_{i}(z)=\left(c_{i} z+d_{i}\right) \mathcal{S}_{q_{1}}\left[K_{x}(1, t)\right](z)-\left(a_{i} z+b_{i}\right) \mathcal{S}_{q_{1}}[K(1, t)](z) .
$$

By Lemma II.1, $\mathcal{S}_{q_{1}}\left[K_{x}(1, t)\right](z)$ and $\mathcal{S}_{q_{1}}[K(1, t)](z)$ are analytic functions of order $1 / 2$. Since the order of a product of analytic functions is the largest of the orders of the factors, c.f. Levin (1980, p. 22], this same property is also inherited by $\Phi_{i}(z)$. Now for each of $i=1,2$, the eigenvalue sequence $z=\left\{\lambda_{n}^{i}\right\}_{n=1}^{\infty}$ forms the zeroes of $\Phi_{i}(z)$ and so from Eq. (15) it follows that both $\Phi_{1}(z)$ and $\Phi_{2}(z)$ must be identically zero. This leads to the pair of equations

$$
\begin{aligned}
& \mathcal{S}_{q_{1}}\left[K_{x}(1, t)\right](z)+\theta_{1}(z) \mathcal{S}_{q_{1}}[K(1, t)](z)=0 \\
& \mathcal{S}_{q_{1}}\left[K_{x}(1, t)\right](z)+\theta_{2}(z) \mathcal{S}_{q_{1}}[K(1, t)](z)=0 .
\end{aligned}
$$

By Eq. (10), the functions $\theta_{1}(z)$ and $\theta_{2}(z)$ are not identically equal and it follows that the analytic functions $\mathcal{S}_{q_{1}}[K(1, t)](z)$ and $\mathcal{S}_{q_{1}}\left[K_{x}(1, t)\right](z)$ must be identically zero. It follows from Lemma II. 1 that $K(1, t)$ and $K_{x}(1, t)$ must also be identically zero. Putting all this together, we have thus shown that if Eq. (10) holds then $K(1, t)=0$ and $K_{x}(1, t)=0$. Lemma II.2 then implies that $q_{1}=q_{2}$. We have proven

Theorem II.1. If the boundary coefficients satisfy the relation

$$
\left|\begin{array}{ll}
a_{1} & a_{2} \\
c_{1} & c_{2}
\end{array}\right| \neq 0 \quad \text { or } \quad\left|\begin{array}{ll}
b_{1} & b_{2} \\
d_{1} & d_{2}
\end{array}\right| \neq 0
$$

and if $\mathcal{L}_{q_{1}}$ and $\mathcal{L}_{q_{2}}$ each have the same spectra corresponding to the boundary conditions

$$
\left(a_{i} \lambda+b_{i}\right) u(1)=\left(c_{i} \lambda+d_{i}\right) u^{\prime}(1), \quad a_{i} d_{i}-b_{i} c_{i}>0, \quad i=1,2,
$$

then $q_{1}=q_{2}$.

Remark 1. The particular bilinear form (8) of the functions $\theta_{i}(z)$ is not critical for this result and a more general boundary dependence on $\lambda$ in Eq. (8) and (9) is clearly 
possible. This would include a fractional polynomial form with a corresponding condition on nontriviality between $\theta_{1}(z)$ and $\theta_{2}(z)$.

\section{GEL'FAND-LEVITAN ALGORITHM}

The uniqueness proof provides a basis for a constructive algorithm similar in nature to that of Rundell and Sacks (1992). Let $q_{1}=0$ and $q_{2}=q$ in the notation of the previous section. $u(x ; q, \lambda)$ satisfies Eq. (6) and has the Gel'fand-Levitan representation

$$
u(x ; q, \lambda)=\frac{\sin \sqrt{\lambda} x}{\sqrt{\lambda}}+\int_{0}^{x} K(x, t) \frac{\sin \sqrt{\lambda} t}{\sqrt{\lambda}} d t
$$

where $K$ satisfies the hyperbolic boundary value problem

$$
\begin{aligned}
& K_{t t}-K_{x x}+q(x) K=0, \quad 0<t<x<1 \\
& K(x, 0)=0, \quad K(x, x)=\frac{1}{2} \int_{0}^{x} q(s) d s .
\end{aligned}
$$

The relationship between $K$ and $q$ can also be expressed as

$$
q(x)=2 \frac{d}{d x} K(x, x)
$$

We use the boundary conditions at $x=1$ from Eq. (9) to uniquely construct Cauchy data for $K$. Then Eq. (20) and the Cauchy data form an overposed boundary value problem for $K$, and Eq. (21) yields $q$.

Let $\left\{\lambda_{n}^{i}\right\}_{n=1}^{\infty}, i=1,2$ be the sequences of eigenvalues corresponding to the boundary condition (9) with $\theta_{i}$ defined by Eq. (8). Evaluating $u$ from Eq. (19) at $x=1$ with $\lambda=\lambda_{n}^{i}$ and applying the appropriate boundary condition yields

$$
\begin{aligned}
& \int_{0}^{1} K_{x}(1, t) \frac{\sin \sqrt{\lambda_{n}^{i}} t}{\sqrt{\lambda_{n}^{i}}} d t-\theta_{i}\left(\lambda_{n}^{i}\right) \int_{0}^{1} K(1, t) \frac{\sin \sqrt{\lambda_{n}^{i}} t}{\sqrt{\lambda_{n}^{i}}} d t \\
& \quad=\theta_{i}\left(\lambda_{n}^{i}\right) \frac{\sin \sqrt{\lambda_{n}^{i}}}{\sqrt{\lambda_{n}^{i}}}-\cos \sqrt{\lambda_{n}^{i}}
\end{aligned}
$$

for $i=1,2$ and $n=1,2, \ldots$ Equations (20)-(22) now form the overdetermined boundary value problem for $K$ mentioned previously. Theorem II.1 shows that the Cauchy data $K(1, t)$ and $K_{x}(1, t)$ can be uniquely recovered from the system (22), and that the Cauchy data uniquely determines the solution of the inverse problem $q$.

Since $q(x)=2(d / d x) K(x, x)$, use of d'Alembert's formula for the inhomogeneous wave equation leads to the following nonlinear integral equation for $q$

$$
q(x)=2\left(K_{t}(1,2 x-1)+K_{x}(1,2 x-1)\right)-2 \int_{x}^{1} q(y) K(y, 2 x-y) d y .
$$

The nonlinearity arises because of the dependence of $K$ on $q$. We can construct $q$ by using a successive approximation method with this integral equation. Choosing an 
initial guess $q_{0}(x)$, set

$$
\begin{aligned}
q_{n+1}(x)= & 2\left(K_{t}(1,2 x-1)+K_{x}(1,2 x-1)\right) \\
& -2 \int_{x}^{1} q_{n}(y) k\left(y, 2 x-y ; q_{n}\right) d y
\end{aligned}
$$

where $k(x, t ; q)$ is the solution of

$$
\begin{aligned}
& k_{t t}-k_{x x}+q(x) k=0, \quad 0 \leq|t| \leq x \leq 1 \\
& k(1, t)=K(1, t), \quad k_{x}(1, t)=K_{x}(1, t), \quad-1 \leq t \leq 1 .
\end{aligned}
$$

The uniqueness of the Cauchy data leads immediately to the unique solution of the boundary value problem (25)-(26) at each step in the successive approximation. Hence each iterate $q_{n+1}$ is uniquely defined by the previous iterate $q_{n}$. The solution of the inverse problem is a fixed point of the mapping $T: q \rightarrow 2(d / d x) k(x, x ; q)$. Uniqueness of the solution of the inverse problem follows since the map $T$ is known to have a unique fixed point, see Rundell and Sacks (1992, Thm. 1).

We can modify the inverse problem so that $\int_{0}^{1} q(x) d x=0$ by estimating $\bar{q}$ from the asymptotics, subtracting it from the eigenvalues and replacing the boundary coefficients $(a, b, c, d)$ with $(a, a \bar{q}+b, c, c \bar{q}+d)$. As a result, it is reasonable to set $q_{0} \equiv 0$. In this case $q_{1}(x)=2\left(K_{t}(1,2 x-1)+K_{x}(1,2 x-1)\right)$ and no solution of the boundary value problem (25)-(26) is computed during the first iteration of the algorithm. $q_{1}(x)$ is simply the linearization of the inverse problem at $q=0$, and in many cases is quite a good approximation to $q$. Using essentially the same argument as Sacks (1988, Lemma 4.1), it can be shown that for $q \in L^{\infty}(0,1)$ it follows that $k \in W^{1, \infty}$. This implies that $q-q_{1} \in C([0,1])$, which is one degree smoother than $q$. Thus $q_{1}$ captures all the discontinuities of $q$, adding to its appeal as an excellent first approximation.

In practice, this algorithm is implemented with finite data. Since infinite sequences of eigenvalues are required for the unique recovery of $q$, one may ask what in fact is being recovered. This algorithm augments the finite data with the eigenvalues of $q_{0}$ that correspond to those missing from our sequences. Essentially, we recover a perturbation from $q_{0}$. Thus the choice of $q_{0}$ is critical - if there are discontinuites in $q$, there should be discontinuities in $q_{0}$.

\section{QUASI-NEWTON ALGORITHM}

Lowe et al. (1992) developed a technique based on a shooting method common in the numerical solution of two-point boundary value problems. For any given potential $q$ and the eigenvalue sequence corresponding to a particular choice of boundary conditions, the eigenfunctions can essentially be determined using only the left endpoint data. Thus the right endpoint boundary condition amounts to additional information which can be used in conjunction with a quasi-Newton scheme to recover the unknown potential $q$.

Recall that the inverse problem is to recover the unknown potential $q \in L^{2}[0,1]$ from two sequences of eigenvalues $\left\{\lambda_{n}^{i}\right\}_{n=1}^{\infty}$ and their associated boundary condition 
constants $a_{i}, b_{i}, c_{i}, d_{i}$, for $i=1,2$, where

$$
\begin{array}{lll}
-u^{\prime \prime}+q u=\lambda_{n}^{1} u, & u(0)=0, & \left(a_{1} \lambda_{n}^{1}+b_{1}\right) u(1)=\left(c_{1} \lambda_{n}^{1}+d_{1}\right) u^{\prime}(1) \\
-u^{\prime \prime}+q u=\lambda_{n}^{2} u, & u(0)=0, & \left(a_{2} \lambda_{n}^{2}+b_{2}\right) u(1)=\left(c_{2} \lambda_{n}^{2}+d_{2}\right) u^{\prime}(1) .
\end{array}
$$

We seek the zeros of the functions

$$
\begin{aligned}
& G_{1}(\lambda ; q)=\theta_{1}(\lambda) u(1 ; q, \lambda)-u^{\prime}(1 ; q, \lambda) \\
& G_{2}(\lambda ; q)=\theta_{2}(\lambda) u(1 ; q, \lambda)-u^{\prime}(1 ; q, \lambda)
\end{aligned}
$$

where, as before, $u(x ; q, \lambda)$ represents the solution to the initial value problem

$$
\mathcal{L}_{q} u:=-u^{\prime \prime}+q(x) u=\lambda u, \quad u(0)=0, \quad u^{\prime}(0)=1
$$

and $\theta_{i}$ are defined by Eq. (8).

We shall seek the approximation

$$
q^{N}(x)=\sum_{k=1}^{2 N} q_{k} \phi_{k}(x)
$$

for which $\lambda_{1}^{i}, \ldots, \lambda_{N}^{i}$ are zeros of the functions $G_{i}\left(\lambda ; q^{N}\right)$ for $i=1,2$. Since we seek $q \in L^{2}[0,1]$, the set $\left\{\phi_{k}(x)\right\}_{k=1}^{2 N}$ should be the first $2 N$ elements of a basis for $L^{2}[0,1]$. In order to remove the necessity of a constant basis function and to facilitate the use of a quasi-Newton technique, we assume here that the data has already been modified so that the potential $q$ has zero mean.

$$
\begin{aligned}
& \text { Let } \Lambda_{N} \equiv\left(\lambda_{1}^{1}, \ldots, \lambda_{N}^{1}, \lambda_{1}^{2}, \ldots, \lambda_{N}^{2}\right) \text {. Let } \vec{q}=\left(q_{1}, \ldots, q_{2 N}\right) \text {. Define } F: R^{2 N} \rightarrow R^{2 N} \text { by } \\
& F_{j}\left(\Lambda_{N} ; \vec{q}\right) \equiv \theta_{1}\left(\lambda_{j}^{1}\right) u_{j}(1 ; \vec{q})-u_{j}^{\prime}(1 ; \vec{q}) \\
& F_{N}+j\left(\Lambda_{N} ; \vec{q}\right) \equiv \theta_{2}\left(\lambda_{j}^{2}\right) v_{j}(1 ; \vec{q})-v_{j}^{\prime}(1 ; \vec{q})
\end{aligned}
$$

for $1 \leq j \leq N$, where $u_{j}(x ; \vec{q})=u\left(x ; q^{N}, \lambda_{j}^{1}\right)$ and $v_{j}(x ; \vec{q})=u\left(x ; q^{N}, \lambda_{j}^{2}\right)$ are defined by Eq. (31). Applying the technique developed by Lowe et al. (1992) for the inverse Sturm-Liouville problem with eigenparameter independent boundary conditions, we seek a vector $\vec{q}=\left(q_{1}, \ldots, q_{2 N}\right)$ for which $F\left(\Lambda_{N} ; \vec{q}\right)=0$.

The use of a quasi-Newton scheme in the eigenparameter independent SturmLiouville problem (Lowe et al., 1992) yields results within $1 \%$ of those attained with a standard Newton scheme. The amount of computation involved in the quasiNewton scheme is significantly lower than in the standard Newton scheme. As a result, we have chosen to implement a quasi-Newton scheme rather than a standard Newton scheme in order to determine $\vec{q}$.

$$
\begin{aligned}
q^{(0)} & =0 \\
F_{q}\left(\Lambda_{N} ; \overrightarrow{0}\right) \delta q^{(m)} & =-F\left(\Lambda_{N} ; q^{(m)}\right) \\
q^{(m+1)} & =q^{(m)}+\delta q^{(m)}
\end{aligned}
$$

where $F_{q}$ is the Jacobian of $F$ and where we have dropped the vector notation on $q$ for convenience. Since $\int_{0}^{1} q(x) d x=0$, the use of $q^{(0)} \equiv 0$ is a reasonable choice. We are essentially linearizing the function $F$ about $q=0$, and provided that $\|q\|$ is not too large, the first iterate gives a good approximation. 
In order to guarantee convergence of the quasi-Newton algorithm (34), the Jacobian $F_{q}\left(\Lambda_{N} ; \overrightarrow{0}\right)$ must be nonsingular. If the Jacobian is well-conditioned, then one can expect accurate results. The rate of convergence of the algorithm depends primarily on $\|q\|$, the distance between the initial guess, $q^{(0)}$, and the function sought, $q$.

The Jacobian $F_{q}\left(\Lambda_{N} ; 0\right)$ has the form

$$
\begin{gathered}
\frac{\partial F_{j}}{\partial q_{k}}\left(\Lambda_{N} ; 0\right)=\theta_{1}\left(\lambda_{j}^{1}\right) \frac{\partial u_{j}}{\partial q_{k}}(1 ; \overrightarrow{0})-\frac{\partial u_{j}^{\prime}}{\partial q_{k}}(1 ; \overrightarrow{0}) \\
\frac{\partial F_{N+j}}{\partial q_{k}}\left(\Lambda_{N} ; 0\right)=\theta_{2}\left(\lambda_{j}^{2}\right) \frac{\partial v_{j}}{\partial q_{k}}(1 ; \overrightarrow{0})-\frac{\partial v_{j}^{\prime}}{\partial q_{k}}(1 ; \overrightarrow{0}) .
\end{gathered}
$$

It is straightforward (Lowe et al., 1992, Lemma 1), to establish that

$$
\frac{\partial u_{j}}{\partial q_{k}}(x ; \overrightarrow{0})=\int_{0}^{x} u_{j}(x-t ; \overrightarrow{0}) u_{j}(t ; \overrightarrow{0}) \phi_{k}(t) d t .
$$

Hence

$$
\begin{aligned}
& \frac{\partial u_{j}}{\partial q_{k}}(1 ; \overrightarrow{0})=\frac{1}{\lambda_{j}^{1}} \int_{0}^{1} \sin \left(\sqrt{\lambda_{j}^{1}}(1-t)\right) \sin \left(\sqrt{\lambda_{j}^{1}} t\right) \phi_{k}(t) d t \\
& \frac{\partial u_{j}^{\prime}}{\partial q_{k}}(1 ; \overrightarrow{0})=\frac{1}{\sqrt{\lambda_{j}^{1}}} \int_{0}^{1} \cos \left(\sqrt{\lambda_{j}^{1}}(1-t)\right) \sin \left(\sqrt{\lambda_{j}^{1}} t\right) \phi_{k}(t) d t
\end{aligned}
$$

with analogous expressions for $\left(\partial v_{j} / \partial q_{k}\right)(1 ; \overrightarrow{0})$ and $\left(\partial v_{j}^{\prime} / \partial q_{k}\right)(1 ; \overrightarrow{0})$.

A block structure for the Jacobian can be ensured by a particular choice of $\left\{\phi_{k}(x)\right\}_{k=1}^{2 N}$. The choice of basis and the asymptotic behavior of the eigenvalues (c.f. Appendix), which is detemined by the nature of the boundary constants $c_{1}, c_{2}$, determine the nature of these blocks. If $c_{1}=0$ and $c_{2} \neq 0$ and the basis

$$
\phi_{k}(x)=\cos (2 k \pi x), \quad \phi_{N+k}=\cos (2 k-1) \pi x \quad k=1, \ldots, N
$$

is used then the Jacobian can be shown to be diagonally dominant provided that the eigenvalues satisfy

$$
\left|\lambda_{j}^{1}-j^{2} \pi^{2}\right| \leq \alpha \pi^{2}, \quad\left|\lambda_{j}^{2}-(j-1 / 2)^{2} \pi^{2}\right| \leq \alpha \pi^{2}
$$

for $\alpha$ sufficiently small. The analysis is similar to that given by Lowe et al. (1992) for their algorithm for the eigenparameter independent inverse Sturm-Liouville problem. By continuity, this diagonal dominance can be extended if the boundary coefficients are varied. However diagonal dominance cannot be proven in general and examples where the Jacobian is not diagonally dominant can be found, e.g., $a_{1}=2, b_{1}=1, c_{1}=0, d_{1}=4$, and $a_{2}=2, b_{2}=1, c_{2}=4, d_{2}=3$ with $q=0$. In our experience, the Jacobian is always nonsingular, but this cannot be proven.

In practice, our choice of basis determines the behavior of the $q$ that we recover. For example, if $q$ is smooth then a Fourier basis is appropriate whereas a basis of linear splines is more appropriate for a piecewise $q$. 


\section{NUMERICAL RESULTS}

The direct problem for a given $q(x)$ and boundary constants $a, b, c, d$ was solved using the FORTRAN software SLEDGE which is specifically designed to solve the eigenparameter dependent Sturm-Liouville problem. Details can be found in (Pruess and Fulton, 1993; Pruess et al., 1995).

The data for the inverse scheme consists of two sets of $N$ eigenvalues and from two different sets of boundary constants. The extra eigenvalue and, if necessary, any negative eigenvalues are removed from the data. In situations where the mean of the potential is non-zero, the mean can be estimated from each sequence using the asymptotic formulae (c.f. Appendix)

$$
\begin{aligned}
& \bar{q}=\lambda_{N}-(N-1 / 2)^{2} \pi^{2}+2 a / c, \quad c \neq 0 \\
& \bar{q}=\lambda_{N}-N^{2} \pi^{2}-2 d / a, \quad c=0
\end{aligned}
$$

as appropriate. The average of these estimates is subtracted from the eigenvalue sequence and used to replace the boundary constants $(a, b, c, d)$ with (a, $a \bar{q}+b, c, c \bar{q}+d)$. The data is then used to reconstruct $q(x)-\bar{q}$, from which the original $q(x)$ is readily obtained. For the Quasi-Newton algorithm, an alternative approach is to ensure that the basis used contains a constant function.

\section{V.A. Implementation Issues for the Gel'fand-Levitan Algorithm}

The Cauchy data $K(1, t), K_{x}(1, t)$, satisfies the two equations represented by Eq. (22). For $i=1,2$ and $n=1,2, \ldots, N$, these equations are of the form

$$
\int_{0}^{1}\left[K_{x}(1, t)-\theta\left(\lambda_{n}^{i}\right) K(1, t)\right] \sin \sqrt{\lambda_{n}^{i}} t d t=f_{i} .
$$

We seek $K(1, t)$ and $K_{x}(1, t)$ of the form

$$
K(1, t)=\sum_{k=1}^{N} \alpha_{k} u_{k}(t), \quad K_{x}(1, t)=\sum_{k=1}^{N} \beta_{k} u_{k}(t)
$$

where $\left\{u_{k}\right\}_{k=1}^{N}$ be a set of basis functions on $[0,1]$. We used $u_{k}(t)=\sin k \pi t$ as our basis and solved the resulting linear system for $\alpha$ and $\beta$. Other choices of $u_{k}$ are feasible, provided that they are chosen so that the matrices in the system remain nonsingular and well-conditioned.

Finally, successive approximation is used in

$$
\begin{aligned}
q_{n+1}(x)= & 2\left(K_{t}(1,2 x-1)+K_{x}(1,2 x-1)\right) \\
& -2 \int_{x}^{1} q_{n}(y) k\left(y, 2 x-y ; q_{n}\right) d y
\end{aligned}
$$

to reconstruct $q$. The reconstructed Cauchy data is used in the first two terms and also in the computation of $k$, which is carried out using a second-order finite difference scheme. As mentioned previously, the choice of $q_{0}$ affects the quality of 
the reconstruction. In all cases discussed, $q_{0} \equiv 0$ was used. The convergence criterion for the algorithm was $\left\|q_{n}-q_{n+1}\right\|_{2}<10^{-4}$.

\section{V.B. Implementation Issues for the Quasi-Newton Algorithm}

Implementation of the Quasi-Newton scheme (34) involves the computation of a nonsingular Jacobian $F_{q}\left(\Lambda_{N} ; \overrightarrow{0}\right)$ and the vector $F\left(\Lambda_{N} ; q^{(m)}\right)$. The computation of $F$ involves the solution of the initial value problems

$$
-u^{\prime \prime}+q(x) u=\lambda u, \quad u(0)=0, \quad u^{\prime}(0)=1
$$

with $q=q^{(m)}$ and $\lambda=\lambda_{i}$ or $\lambda=\mu_{i}$ as appropriate. This was carried out using a Runge-Kutta adaptive routine from the Matlab ODE suite. Although a variety of different bases were tested, the basis used in the folowing examples was

$$
\phi_{k}(x)=\cos (2 k \pi x), \phi_{N+k}=\cos (2 k-1) \pi x \quad k=1, \ldots, N .
$$

The convergence criterion for the algorithm was $\|\delta q\|_{2}<10^{-4}$.

\section{V.C. Examples}

The first example considers the function $q(x)=16 x^{2} e^{-8 x}-\left(1-41 e^{-8}\right) / 16$ and the boundary conditions associated with the constants $a_{1}=2, b_{1}=2, c_{1}=0, d_{1}=1$ and $a_{2}=2, b_{2}=1, c_{2}=1, d_{2}=1$. This function has zero mean, $\bar{q}=0$. Figure 1 shows the application of the Quasi-Newton algorithm to $q$ using sequences of 5 and 10 eigenvalues. Both reconstructions converged in 3 iterations with residuals of $\left\|q-q^{5}\right\|_{2}=0.0099$, and $\left\|q-q^{10}\right\|_{2}=0.0039$. Figure 2 shows the application of the Gel'fand-Levitan algorithm to $q_{1}$ using sequences of 5 and 10 eigenvalues. Both reconstructions converged in 2 iterations with residuals of $\left\|q-q^{5}\right\|_{2}=0.0201$, and $\left\|q-q^{10}\right\|_{2}=0.0089$.

Figure 3 shows the results of both algorithms for the piecewise function

$$
q(x)= \begin{cases}14 x-5 & 0<x \leq 1 / 2 \\ 2 & 1 / 2<x \leq 3 / 4 \\ 1 & 3 / 4<x \leq 1\end{cases}
$$

With sequences of 10 eigenvalues, the Quasi-Newton algorithm converged in 7 iterations with a residual of $\left\|q-q^{7}\right\|_{2}=0.8543$. Most of the error occurs at the discontinuity. This is due to the fact that we are essentially constructing a Fourier series which, at a discontinuity, will converge to average of the right-handed and lefthanded limits. With sequences of 10 eigenvalues, the Gel'fand-Levitan algorithm also converged in 7 iterations but with a residual of $\left\|q-q^{7}\right\|_{2}=7.5712$. For piecewise functions, this algorithm requires far more eigenvalues to produce accurate results. With sequences of 44 eigenvalues, the residual was $\left\|q-q^{6}\right\|_{2}=1.4049$ after 6 iterations. A piecewise choice of $q_{0}$ would improve this. 

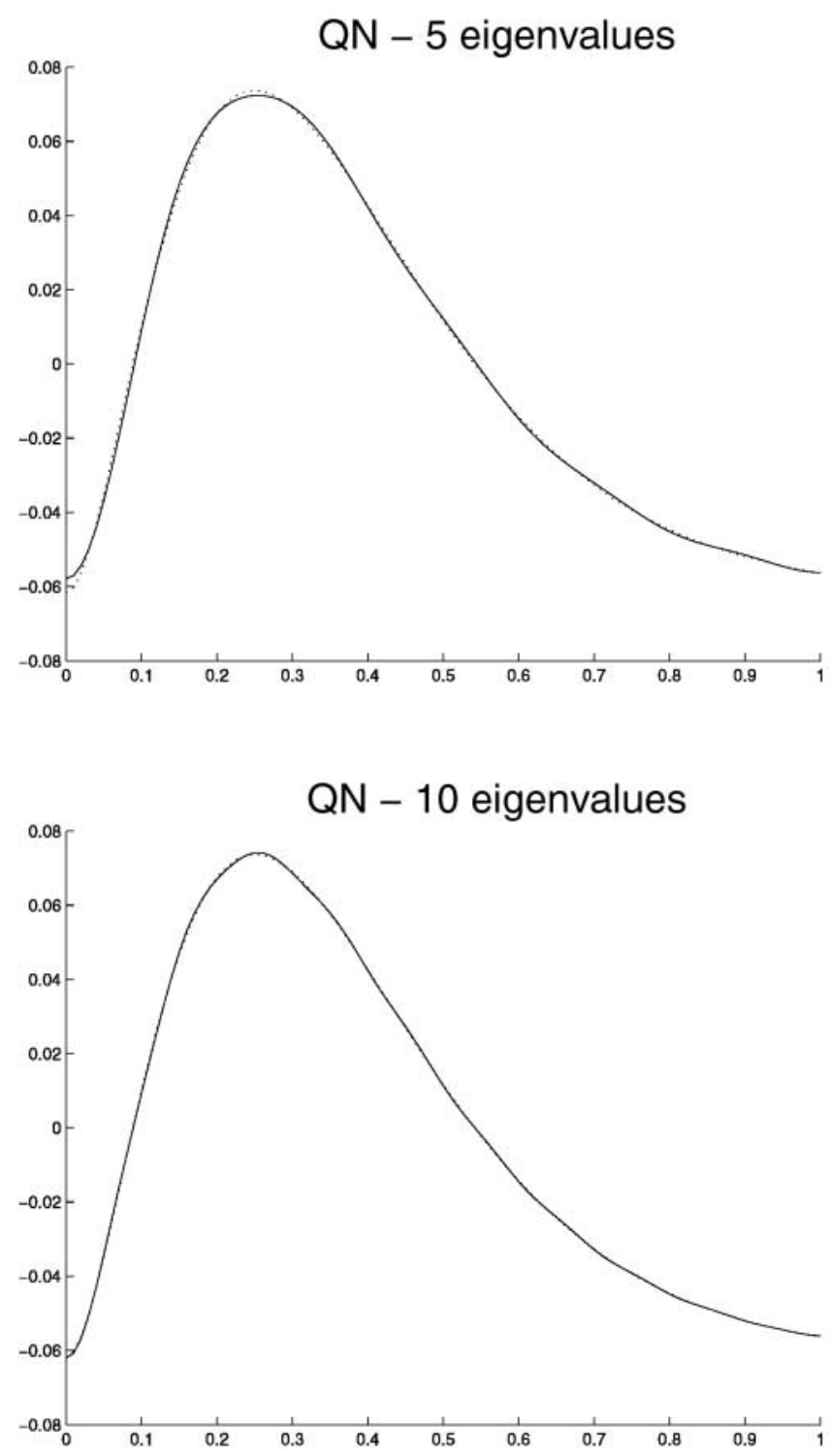

Figure 1. Quasi-Newton algorithm applied to $q(x)=16 x^{2} e^{-8 x}-\left(1-41 e^{-8}\right) / 16$ from boundary conditions associated with $a_{1}=2, b_{1}=2, c_{1}=0, d_{1}=1$ and $a_{2}=2, b_{2}=1$, $c_{2}=1, d_{2}=1$.

Figure 4 shows $q(x)=6 x^{2}(1-x)$, which has nonzero mean, $\bar{q}=0.5$. The reconstructions shown are for boundary constants $a_{1}=2, b_{1}=2, c_{1}=0, d_{1}=1$ and $a_{2}=2, b_{2}=1, c_{2}=1, d_{2}=1$, with 10 eigenvalues. For the Quasi-Newton algorithm, the reconstruction converged in 3 iterations with a residual $\left\|q-q^{10}\right\|_{2}=0.0889$. For the Gel'fand-Levitan algorithm, the reconstruction converged in 3 iterations with a residual $\left\|q-q^{10}\right\|_{2}=0.0766$. 

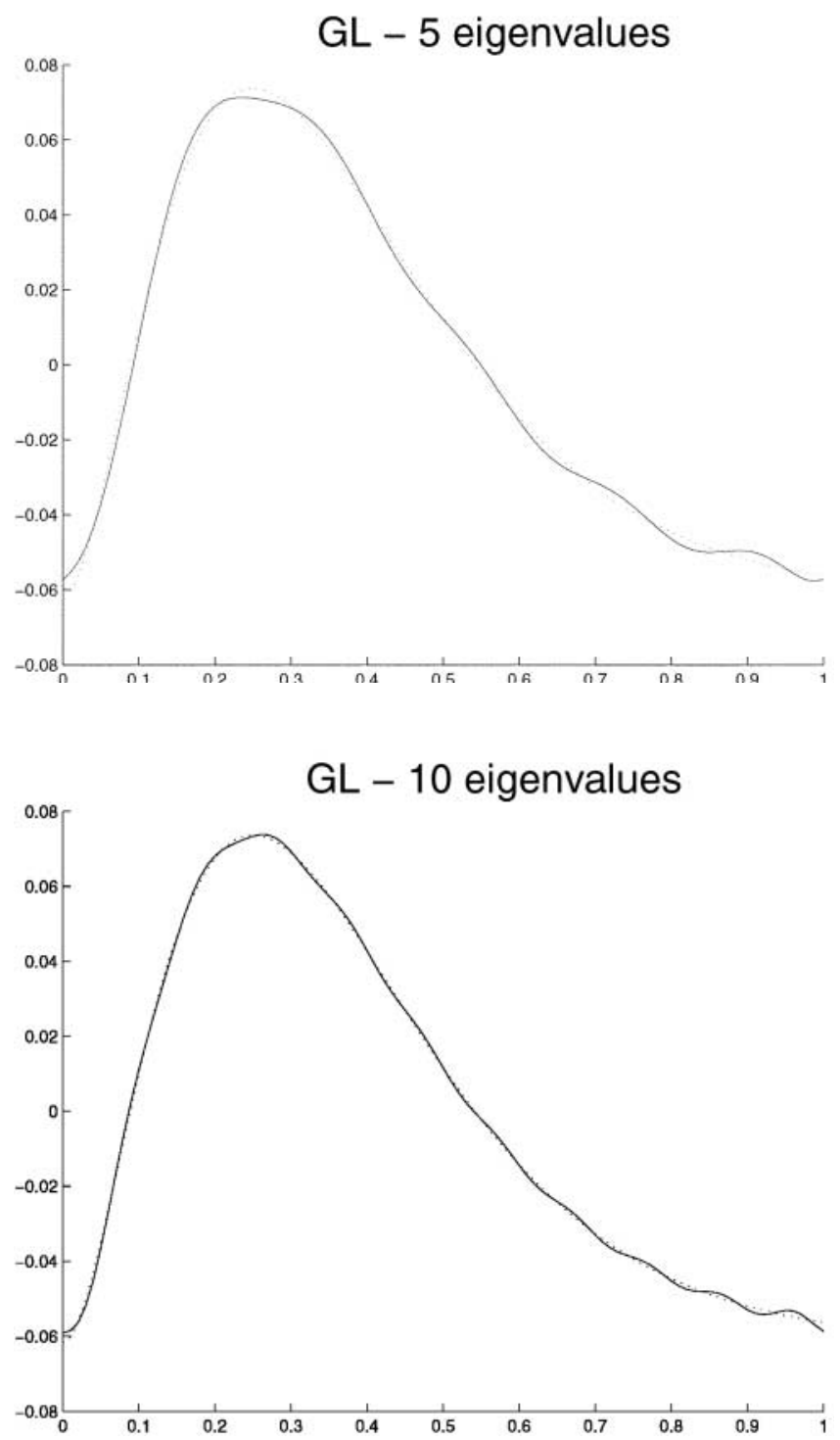

Figure 2. Gel'fand-Levitan algorithm applied to $q(x)=16 x^{2} e^{-8 x}-\left(1-41 e^{-8}\right) / 16$ from boundary conditions associated with $a_{1}=2, b_{1}=2, c_{1}=0, d_{1}=1$ and $a_{2}=2, b_{2}=1$, $c_{2}=1, d_{2}=1$.

\section{V.C.1. Variation of the Boundary Constants}

From the uniqueness argument of Sec. II, our boundary constants must satisfy either

$$
\left|\begin{array}{ll}
a_{1} & a_{2} \\
c_{1} & c_{2}
\end{array}\right| \neq 0 \quad \text { or } \quad\left|\begin{array}{ll}
b_{1} & b_{2} \\
d_{1} & d_{2}
\end{array}\right| \neq 0
$$



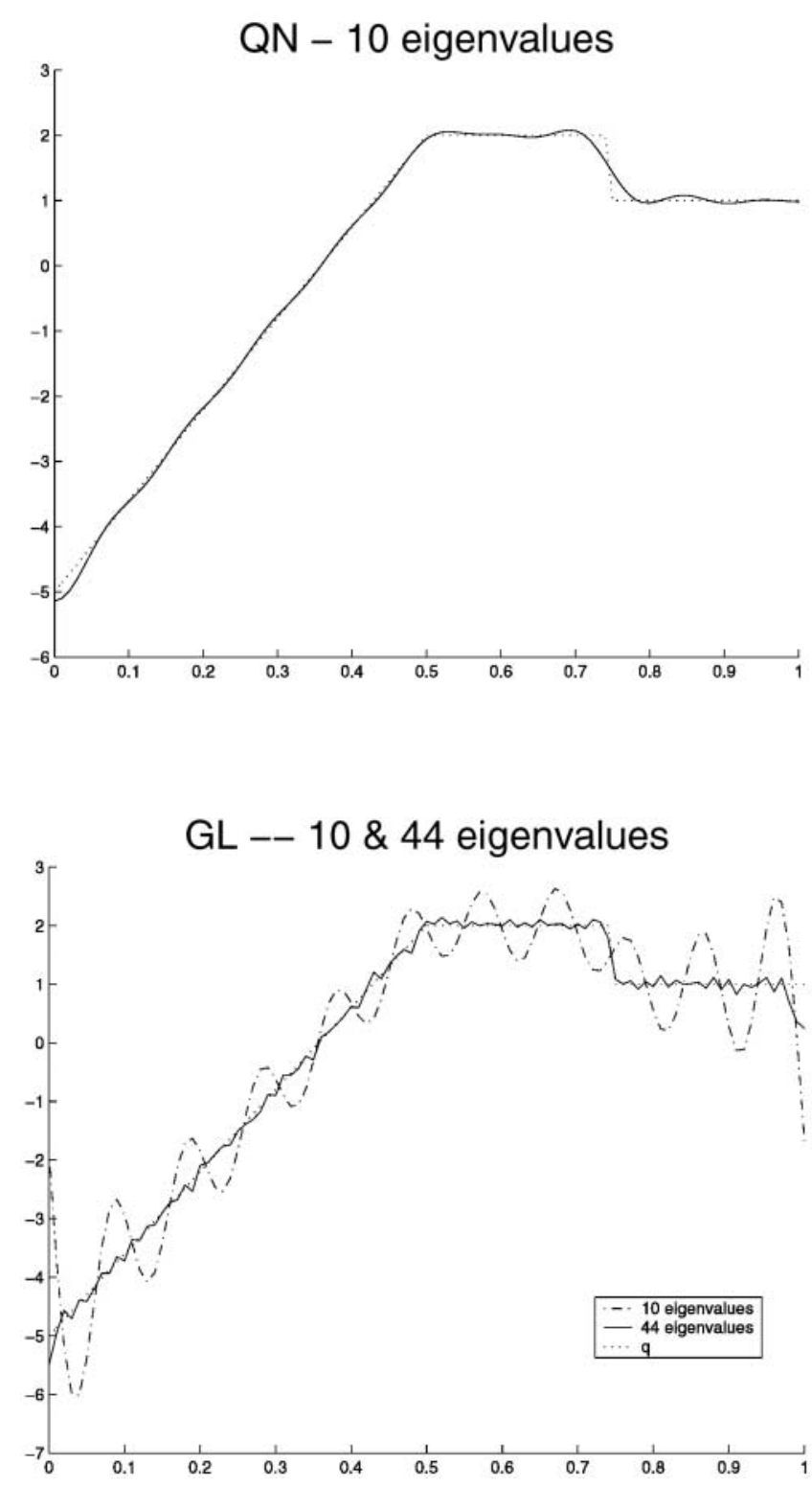

Figure 3. Quasi-Newton and Gel'fand-Levitan algorithms applied to piecewise $q(x)$ from boundary conditions associated with $a_{1}=2, b_{1}=2, c_{1}=0, d_{1}=1$ and $a_{2}=2, b_{2}=1$, $c_{2}=1, d_{2}=1$.

in order for a unique solution of the inverse problem to exist. These conditions will be referred to as the $a c$-condition and the $b d$-condition.

Figure 5 shows reconstructions from the quasi-Newton algorithm for various boundary conditions. Figure 6 shows reconstructions from the Gel'fand-Levitan algorithm. In both cases, $q(x)=6 x^{2}(1-x)-1 / 2$ is recovered from data sequences 

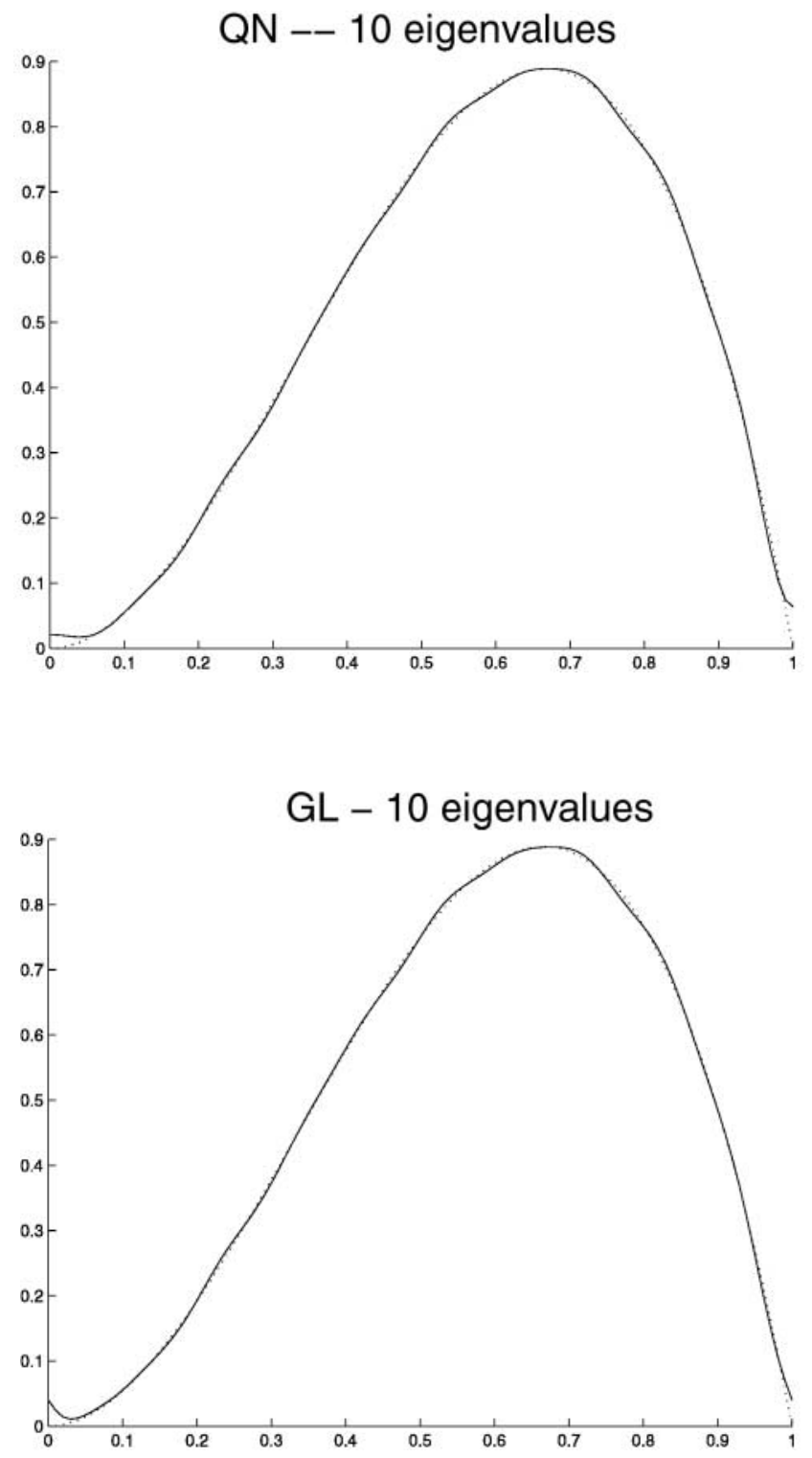

Figure 4. Quasi-Newton and Gel'fand-Levitan algorithms applied to $q(x)=6 x^{2}(1-x)$ from boundary conditions associated with $a_{1}=2, b_{1}=2, c_{1}=0, d_{1}=1$ and $a_{2}=2, b_{2}=1$, $c_{2}=1, d_{2}=1$.

of 10 eigenvalues, whose boundary constants satisfy either the $a c$-condition (top right), the $b d$-condition (bottom left and right) or both conditions (top left). The top left graphs in Figs. 5 and 6 have boundary constants that satisfy both the $a c$ condition and the $b d$-condition. The $a c$-condition is violated in the top right and 
bottom left graphs. The $b c$-condition is violated in the bottom right graphs. It is worth noting that the most significant degradation in the quality of the reconstruction occurs when when the violation of the $a c$-condition is due to $c_{1}=c_{2}=0$. In this case, the asymptotic behavior of the two eigenvalue sequences is so similar that the algorithms cannot differentiate between them well enough to build a good reconstruction.
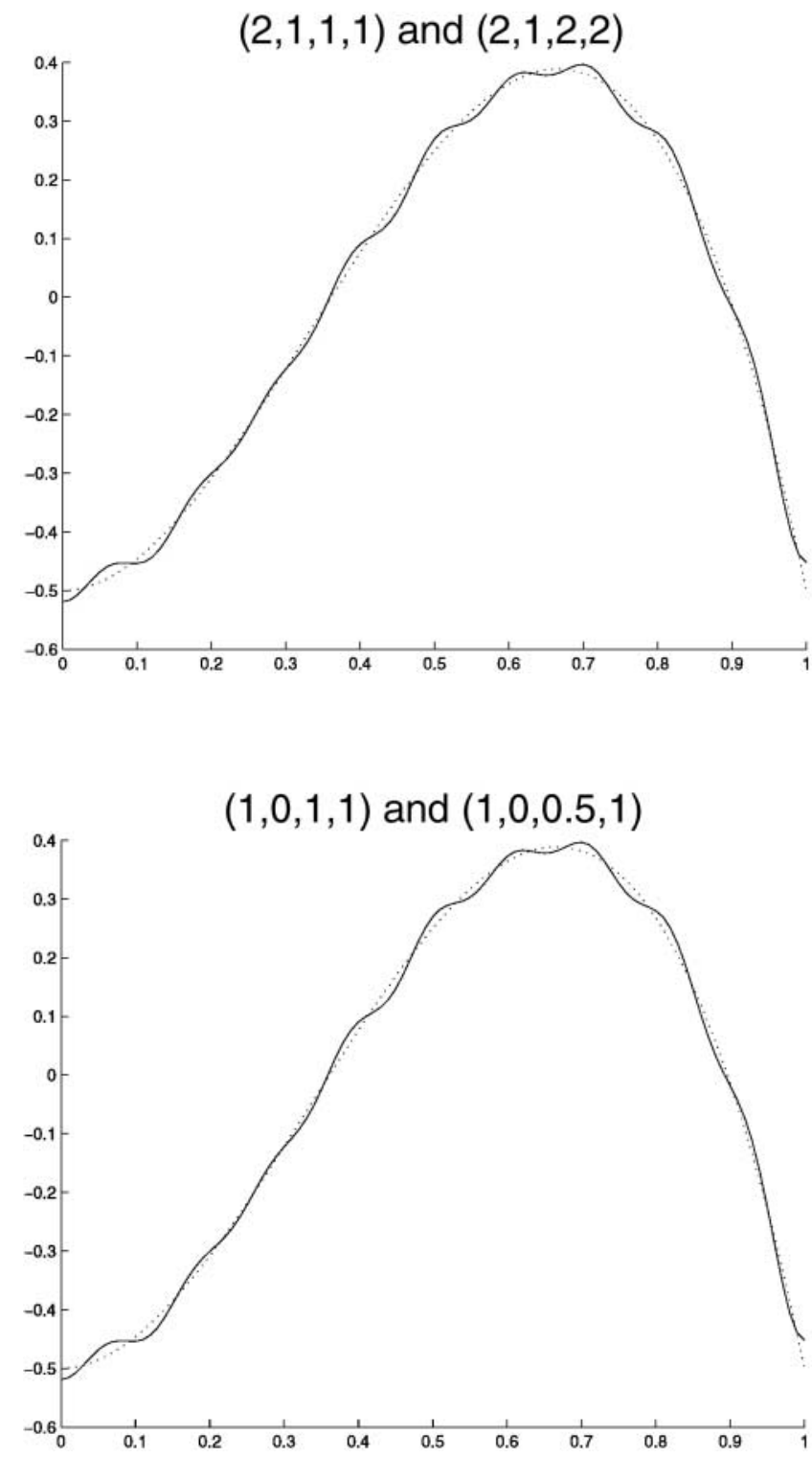

Figure 5. Quasi-Newton recovery of $q$ from various combinations of boundary conditions. 

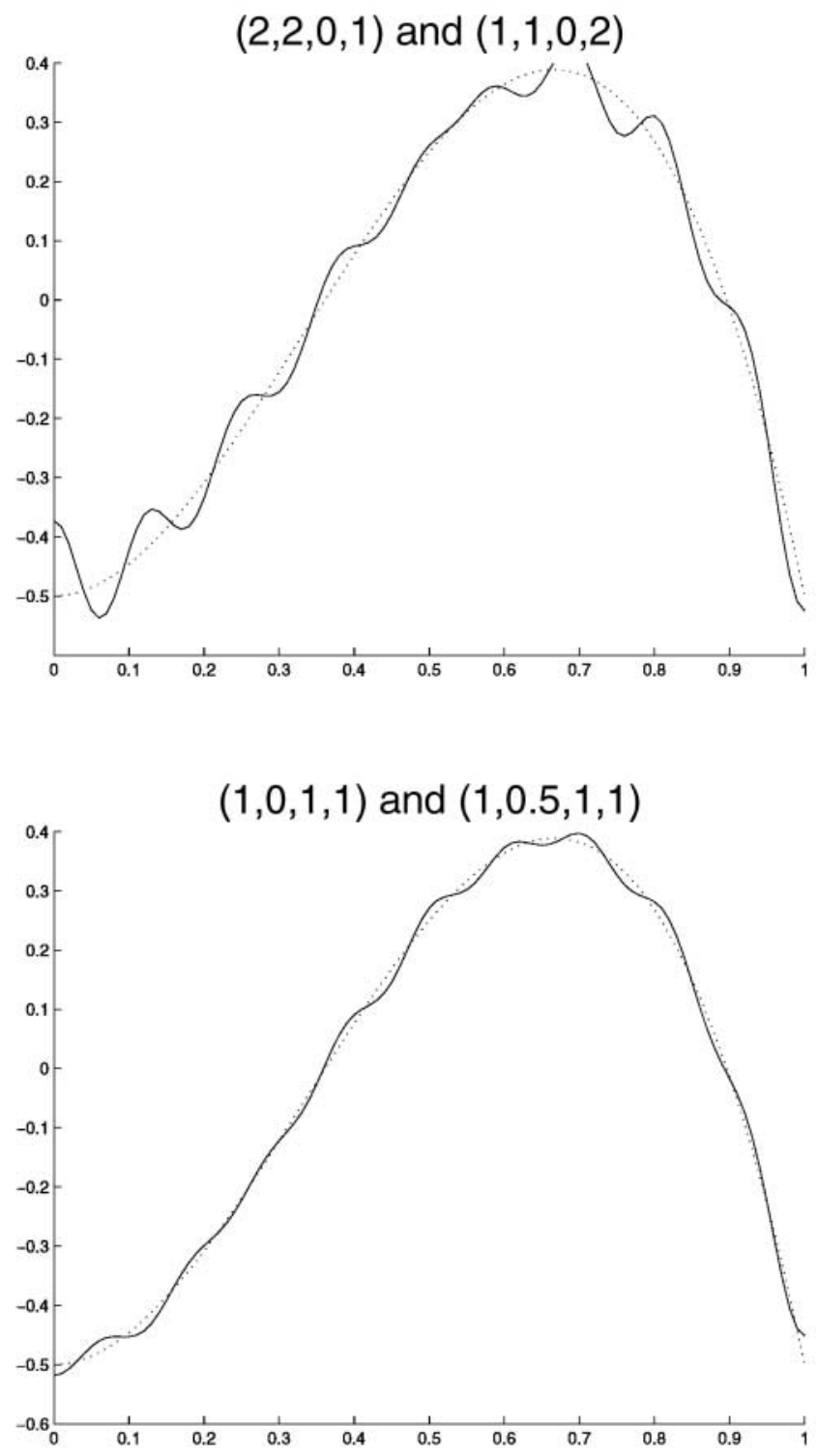

Figure 5. Continued.

\section{APPENDIX. ASYMPTOTIC BEHAVIOR OF THE EIGENVALUES}

Let $\lambda_{n}^{D}, n=1,2,3, \ldots$ be the eigenvalues of the problem with Dirichlet boundary conditions at both ends,

$$
-u^{\prime \prime}+q u=\lambda u, \quad u(0)=0, \quad u(1)=0 .
$$



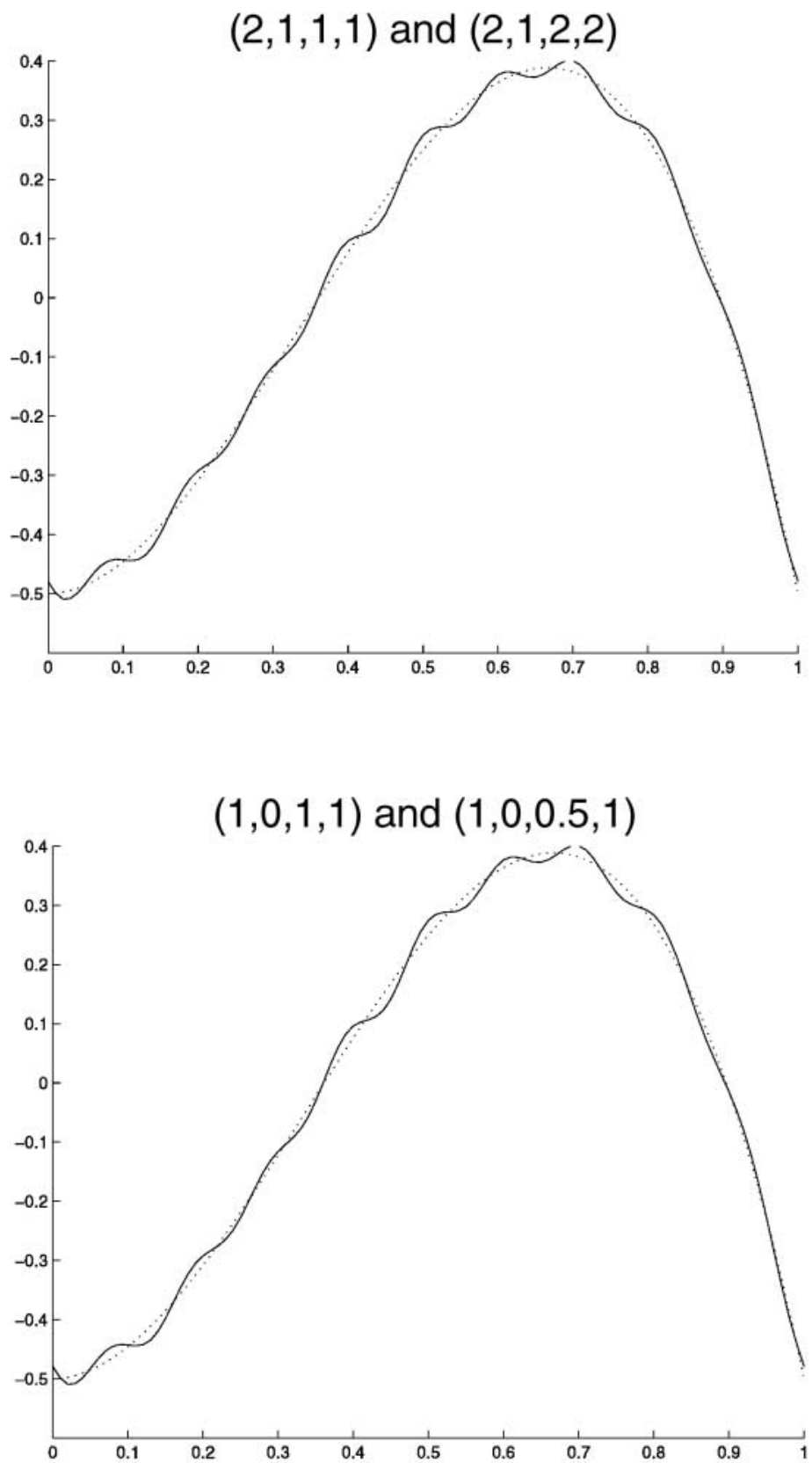

Figure 6. Gel'fand-Levitan recovery of $q$ from various combinations of boundary conditions. 

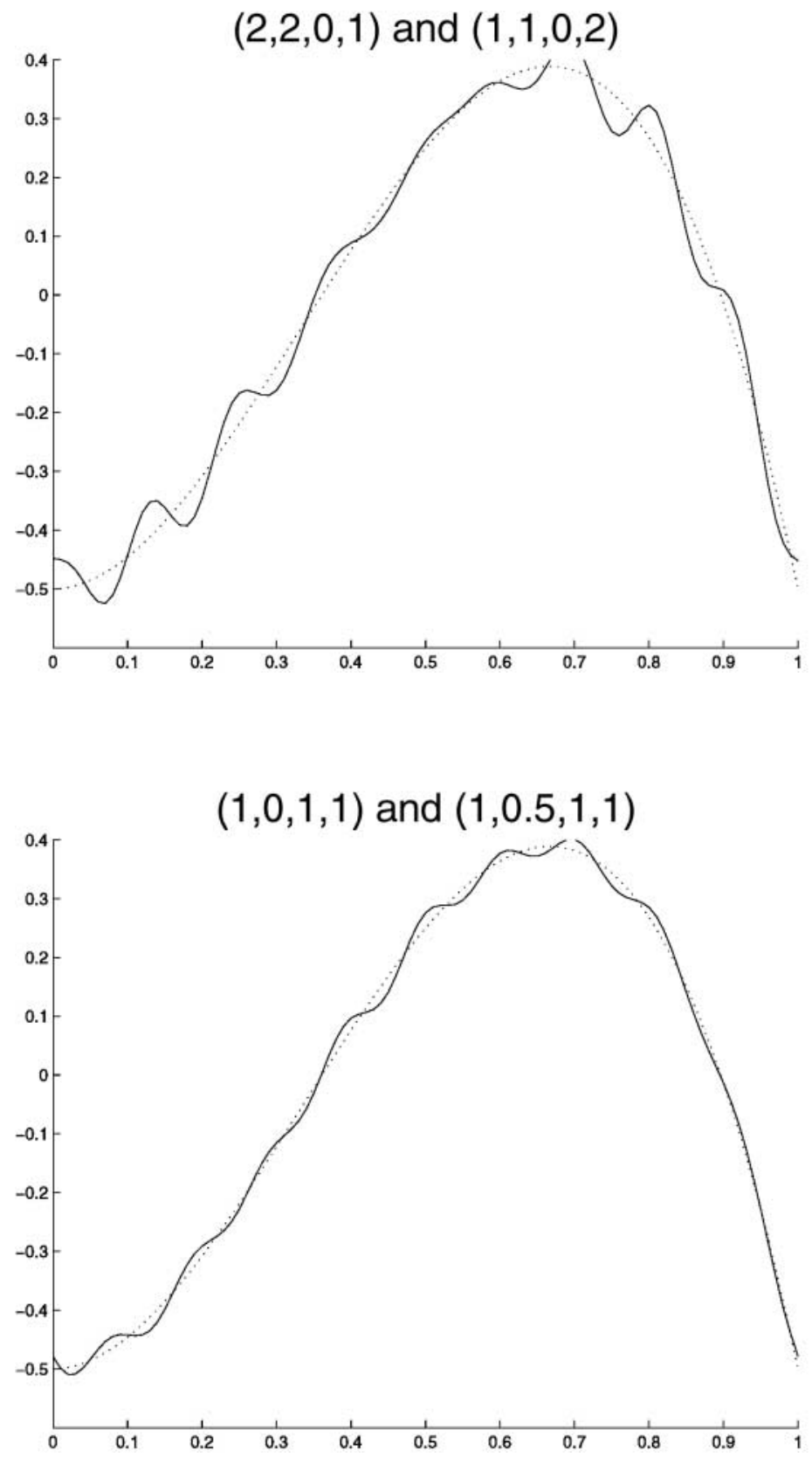

Figure 6. Continued. 
Theorem A.1. If $\delta=a d-b c>0$ and $\bar{q}=\int_{0}^{1} q$, then the eigenvalues of $(1,2,3)$ are given by $\lambda^{\prime}$ and the sequence $\lambda_{1}<\lambda_{2}<\lambda_{3} \cdots$.

- Dirichlet-Dirichlet eigenvalues: If $c=0$, then $\lambda^{\prime}<\lambda_{1}$, the eigenfunctions $u_{n}$ corresponding to $\lambda_{n}$ have exactly $n$ zeros in $(0,1)$ and

$$
\sqrt{\lambda_{n}}=n \pi+\frac{1}{n \pi}\left\{\frac{\bar{q}}{2}+\frac{d}{a}\right\}+O\left(\frac{1}{n^{2}}\right) .
$$

That is

$$
\lambda_{n}=n^{2} \pi^{2}+\bar{q}+2 d / a+o(1) .
$$

- Dirichlet-Neumann eigenvalues: If $c \neq 0$, the eigenfunctions $u_{n}$ corresponding to $\lambda_{n}$ have exactly $n-1$ zeros in $(0,1)$ and

$$
\sqrt{\lambda_{n}}=(n-1 / 2) \pi+\frac{1}{(n-1 / 2) \pi}\left\{\frac{\bar{q}}{2}-\frac{a}{c}\right\}+O\left(\frac{1}{n^{2}}\right) .
$$

That is

$$
\lambda_{n}=(n-1 / 2)^{2} \pi^{2}+\bar{q}-2 a / c+o(1) .
$$

If $N \geq 0$ is such that $\lambda_{N}^{D}<-d / c \leq \lambda_{N+1}^{D}$, where $\lambda_{0}^{D}=-\infty$, then the eigenfunction corresponding to $\lambda^{\prime}$ has exactly $N$ zeros in $(0,1)$.

This theorem is essentially a restatement of Theorems 3.1, 3.5, and 5.3 and Corollaries 3.6 and 5.4 from Binding et al. (2000), although the notation has been modified to that of Browne and Sleeman (1997). Extensive discussion of the spectral theory of this type of problem can be found in various articles of Binding et al. (1993, 2000, 2002), and Browne and Sleeman (1996).

\section{ACKNOWLEDGMENT}

The first author gratefully acknowledges partial support from the Association for Women in Mathematics, the Kentucky National Science Foundation Experimental Program for the Stimulation of Competitive Research (KY NSF EPSCoR) and the National Science Foundation. The second author gratefully acknowledges partial support from the National Science Foundation.

\section{REFERENCES}

Binding, P. A., Browne, P. J., Seddighi, K. (1993). Sturm-Liouville problems with eigenparameter dependent boundary condtions. Proceedings of the Edinburgh Mathematical Society 37:57-72.

Binding, P. A., Browne, P. J., Watson, B. A. (2000). Inverse spectral problems for Sturm-Liouville equations with eigenparameter eependent boundary conditions. J. London Mathematical Society 62:161-182. 
Binding, P. A., Browne, P. J., Watson, B. A. (2002). Spectral isomorphisms between generalized Sturm-Liouville problems. Linear Operators and Matrices. Birkhäuser, pp. 135-152; Oper. Theory Adv. Appl. 130.

Browne, P. J., Sleeman, B. D. (1996). Inverse nodal problems for Sturm-Liouville equations with eigenparameter dependent boundary conditions. Inverse Problems 12:377-381.

Browne, P. J., Sleeman, B. D. (1997). A uniqueness theorem for inverse eigenparameter dependent Sturm-Liouville problems. Inverse Problems 13:1453-1462.

Fulton, C. T. (1977). Two-point boundary value problems with eigenvalue parameter contained in the boundary conditions. Proc. Royal Soc. Edin. 77A:293-308.

Levin, B. Ja. (1980). Distribution of zeros of entire functions. Translations of Mathematical Monographs. Vol. 5. AMS.

Lowe, B., Pilant, M., Rundell, W. (1992). The recovery of potentials from finite spectral data. SIAM J. Math. Anal. 23(2):482-504.

Pöschel, J., Trubowitz, E. (1987). Inverse spectral theory. Pure and Applied Mathematics. Vol. 130. Academic Press.

Pruess, S., Fulton, C. T. (1993). Mathematical software for Sturm-Liouville problems. ACM Trans. on Math. Software 19:360-376.

Pruess, S., Fulton, C.T., Xie, Y. (1995). An asymptotic numerical method for a class of singular Sturm-Liouville problems. SIAM J. Numer. Anal. 32:1658-1676.

Rundell, W., Sacks, P. E. (1992). Reconstruction techniques for classical inverse Sturm-Liouville Problems. Mathematics of Computation 58(197):161-183.

Sacks, P. E. (1988). An iterative method for the inverse Dirichlet problem. Inverse Problems 4:1055-1069.

Walter, J. (1973). Regular eigenvalue problems with eigenvalue parameter in the boundary condition. Math. Z. 133:301-312. 


\section{MARCEl DeKKer, INC. • 270 MAdison AVENUE • New York, NY 10016}

C2003 Marcel Dekker, Inc. All rights reserved. This material may not be used or reproduced in any form without the express written permission of Marcel Dekker, Inc. 
Copyright $\odot 2003$ EBSCO Publishing 